\title{
Not all $\beta$-lactams are equal regarding neurotoxicity
}

\author{
Khalil Chaïbi ${ }^{1}$, Maïté Chaussard ${ }^{1,2}$, Sabri Soussi ${ }^{1,2}$, Matthieu Lafaurie ${ }^{4}$ and Matthieu Legrand ${ }^{1,2,3^{*}}$
}

See related research by May et al. https://ccforum.biomedcentral.com/articles/10.1186/s13054-016-1394-2

We read with interest the letter from May et al. recently published in Critical Care [1]. The authors suggest that overdosing of $\beta$-lactams in 108 critically ill patients receiving renal replacement therapy may not be associated with neurotoxicity. We agree that adequate plasmatic levels of $\beta$-lactams in critically ill patients with sepsis should be a primary goal and that under-dosing may put the patient at a dangerous risk of treatment failure [2]. We are, however, concerned about the conclusions of this letter and would like to comment on two points.

First, not all $\beta$-lactams are equal regarding neurotoxicity, and some may expose the patient to higher risk. A number of studies have reported neurotoxicity of imipenem, especially in patients with brain injury $[3,4]$. More recently, several authors have reported neurotoxicity with cefepime. Fugate et al. reported that $7 \%$ to $15 \%$ of critically ill patients treated with cefepime developed definitive or possible neurotoxicity [5]. Hence, renal insufficiency was a major risk factor. We have also observed over the last 2 years similar findings with four cases of cefepime-related neurotoxicity in our intensive care unit (ICU) in patients with chronic renal insufficiency or acute kidney injury.

Second, May et al. defined neurotoxicity as an association of overdose and convulsions. There is, however, strong evidence showing that neurotoxicity of $\beta$-lactams can present without seizures [6]. Cefepime-related neurotoxicity has been reported to present mostly as myoclonus and impaired consciousness, confusion, hallucinations, or agitation. Convulsions might be only the most extreme manifestation of the induced encephalopathy toxicity. In our cases, the neurological manifestations were loss of contact, agitation, disorientation and, for two of them, myoclonic twitches and jerks. The observed cefepime trough level of those patients during these events ranged from $144 \mathrm{mg} / \mathrm{l}$ to $455 \mathrm{mg} / \mathrm{l}$ for an upper therapeutic trough level of $90 \mathrm{mg} / \mathrm{l}$ according to our laboratory, while daily doses were 4 to $6 \mathrm{~g} /$ day. An electroencephalogram was carried out for each patient and has objectified a toxic encephalopathy pattern or aspecific slow waves with no sign of lobe epilepsy. Every patient experienced a regression of the symptoms with the withdrawal of the offending drug.

To conclude, while we agree that high serum levels of $\beta$-lactams should be obtained at the initiation of antibiotic therapy in patients with sepsis, intensivists should not overlook the potential neurotoxicity of some $\beta$ lactams with a low therapeutic index such as imipenem and cefepime. The risk of cefepime neurotoxicity should not be underappreciated in patients with neurological symptoms, especially if renal function is impaired.

\section{Authors' response}

Faten May, Najouah El-Helali, Jean-François Timsit and Benoît Misset

We thank Dr Chaïbi et al. for their valuable and insightful comments on our report of the low evidence for an association between supra-therapeutic serum levels of $\beta$-lactams (BL) and clinical toxicity in ICU

\footnotetext{
* Correspondence: matthieu.legrand@aphp.fr

${ }^{1}$ Department of Anesthesiology and Critical Care and Burn Unit, St-Louis

Hospital, Assistance Publique- Hopitaux de Paris, Paris, France

${ }^{2}$ University Paris Diderot, Paris, France

Full list of author information is available at the end of the article
}

patients with acute renal failure (ARF) treated with intermittent hemodialysis [1].

Chaïbi et al. point out that neurotoxicity is common among BL, especially in at-risk patients such as those with ARF, and that some BL with a low therapeutic index may induce more neurological events. Several reports described an increased risk for penicillins, imipenem, and fourth generation cephalosporins, and suggested that neurological complications may range from 
confusion, depressed level of consciousness, and nonspecific encephalopathy to myoclonus, non-convulsive status epilepticus (NCSE), and seizures [6].

We would like to draw attention to the fact that the majority of those articles were non-human experimental studies, case series, and retrospective reviews, with assessment offering low levels of evidence [7]. To establish a cause-effect relationship between a BL administration and clinical signs of encephalopathy remains a major difficulty because both clinical and electrical signs are non-specific. All the neurological symptoms of clinical encephalopathy may be observed in conditions such as metabolic abnormality, uremic disorder, ICU delirium, withdrawal syndrome, or septic encephalopathy, which are very frequent in ICU septic patients [8].

In our population, while we may have missed nonconvulsive signs and NCSE, we could not find an association between convulsions and $\mathrm{BL}$ observed at the supra-therapeutic level.

Therefore, despite the comments of Chaïbi et al., we consider that the BL neurotoxic threshold is not yet well identified and is still challenging. A proper diagnosis of BL neurological toxicity may be obscured by the overall clinical picture and may easily be attributed to the infectious process or to an underlying metabolic. We estimate that prospective observational studies, including pre-established and precise definitions and design, are still needed to assess BL encephalopathy and the neurotoxic threshold and to conclude on their clinical impact.

\footnotetext{
Abbreviations

BL: Beta-lactams; ICU: Intensive care unit; ARF: Acute renal failure; NCSE: Non-
} convulsive status epilepticus

\section{Availability of data and materials}

Yes.

\section{Authors' contribution}

KC and ML wrote the first draft of the manuscript. All authors read and approved the final manuscript.

\section{Competing interests}

The authors declare that they have no competing interests.

\section{Author details}

'Department of Anesthesiology and Critical Care and Burn Unit, St-Louis Hospital, Assistance Publique- Hopitaux de Paris, Paris, France. ${ }^{2}$ University Paris Diderot, Paris, France. ${ }^{3}$ UMR INSERM 942, Institut National de la Santé et de la Recherche Médicale (INSERM), Lariboisière Hospital University Paris Diderot, F-75475 Paris, France. ${ }^{4}$ AP-HP, Infectious disease, St-Louis Hospital, Paris, France.

Published online: 27 October 2016

\section{References}

1. May F, El-Helali N, Timsit J-F, Misset B. Absence of obvious link between supra-therapeutic serum levels of $\beta$ lactams and clinical toxicity in ICU patients with acute renal failure treated with intermittent hemodialysis. Crit Care. 2016:20:220

2. Honore PM, Spapen HD. Cefepime-induced neurotoxicity in critically ill patients undergoing continuous renal replacement therapy: beware of dose reduction! Crit Care. 2015;19:455.
3. Chapuis TM, Giannoni E, Majcherczyk PA, Chioléro R, Schaller M-D, Berger MM, et al. Prospective monitoring of cefepime in intensive care unit adult patients. Crit Care. 2010;14:R51.

4. Sutter R, Rüegg S, Tschudin-Sutter S. Seizures as adverse events of antibiotic drugs: a systematic review. Neurology. 2015;85:1332-41.

5. Fugate JE, Kalimullah EA, Hocker SE, Clark SL, Wijdicks EFM, Rabinstein AA. Cefepime neurotoxicity in the intensive care unit: a cause of severe, underappreciated encephalopathy. Crit Care. 2013;17:R264.

6. Lamoth F, Buclin T, Pascual A, Vora S, Bolay S, Decosterd LA, Calandra T, Marchetti $O$. High cefepime plasma concentrations and neurological toxicity in febrile neutropenic patients with mild impairment of renal function. Antimicrob Agents Chemother. 2010;54(10):4360-7.

7. Grill MF, Maganti RK. Neurotoxic effects associated with antibiotic use: management considerations. Br J Clin Pharmacol. 2011;72(3):381-93.

8. Bhattacharyya S, Darby RR, Raibagkar P, Gonzalez Castro LN, Berkowitz AL. Antibiotic-associated encephalopathy. Neurology. 2016;86(10):963-71. 